\title{
Formación inicial y percepción del profesorado sobre los estilos de enseñanza en Educación Física Teachers' education and perception of teaching styles in Physical Education María Fernández Rivas, María Espada Mateos Universidad Camilo José Cela (España)
}

\begin{abstract}
Resumen. Los estilos de enseñanza son una herramienta metodológica importante de aplicación en las clases de Educación Física. En la presente investigación se pretende conocer cómo es la formación inicial y percepción a la hora de usar los estilos de enseñanza por parte de los profesores de Educación Física en función del sexo, la edad y la titulación de éstos. Para ello, se ha utilizado una muestra de 455 profesores de Educación Física (70,8\% de hombres y 29,2\% de mujeres) con un rango de edad desde 23 a 63 años (M=38,47; DT=8,435) de la Comunidad de Madrid. El análisis de los datos se ha llevado a cabo gracias un análisis descriptivo y un análisis inferencial (t-Student, ANOVA y Welch). Los resultados más significativos muestran que son los profesores de entre 31 y 50 años los que consideran más difícil la aplicación de los estilos de enseñanza en mayor medida que los profesores más jóvenes, con edades comprendidas entre 23-30 años (31-40, $\mathrm{p}<.001 ; 41-50, \mathrm{p}=.039)$. Los docentes con edades menores de 40 años $(<30$, $\mathrm{p}=.02$; 31$40, \mathrm{p}=.014$ ) son los que consideran que se han desempeñado mejor a la hora de aplicar los estilos de enseñanza que aquellos profesores mayores de 51 años. Por último, tanto los licenciados o graduados en Ciencias de Actividad Física y Deporte ( $\mathrm{p}=.008)$ como los profesores que además son diplomados o graduados en magisterio de Educación Física $(\mathrm{p}=.01$ ) consideran que su formación práctica fue buena, al contrario que los diplomados o graduados en magisterio de Educación Física.
\end{abstract}

Palabras clave: Estilos de enseñanza, Educación Física, Percepción, Formación inicial.

Abstract. Teaching styles are an important methodological tool for carrying out Physical Education (PE) classes. This research aims to understand the relation between PE teachers' education and perceptions and their use of teaching styles by gender, age and qualification. A sample of 455 PE teachers (70.8\% men and 29.2\% women) from the Community of Madrid, with age ranged from 23 to 63 years (M=38.47; DT=8.435), was selected for these purposes. Descriptive and inferential analysis (Student's t, ANOVA and Welch) were run for analysing the data collected. The most significant results show that teaching styles are more difficult to be used by teachers are 31 to 50 years old than by younger ones, aged between 23-30 (31-40, $<<.001$; 41-50, $\mathrm{p}=.039$ ). Teachers younger than 40 years old have higher consideration of their use of teaching styles than those aged 51 or older $(<30$, $\mathrm{p}=.02$; 31-40, $\mathrm{p}=.014)$. Finally, graduates in Physical Activity and Sport Sciences $(\mathrm{p}=.008)$ and teachers who have an additional diploma of Physical Education teaching $(\mathrm{p}=.01)$ believe that their internship was useful, whereas teachers who only hold a diploma of Physical Education teaching do not share this perception.

Keywords: Teaching styles, Physical Education, Perception, Education.

\section{Introducción}

Para poder tratar los estilos de enseñanza es necesario conocer y clarificar algunos conceptos de las Ciencias de la Educación como son modelo, método y estilo de enseñanza.

Así, Metzler (2005) habla de enseñanza basada en el modelo, definiéndola como aquella educación en la que tanto el contenido como los estilos de enseñanza deben estar relacionados con los resultados planteados, teniendo en cuenta a la hora de aplicar los estilos de enseñanza la experiencia, las características de los alumnos y el contexto donde se da el aprendizaje. Así, plantea 8 modelos diferentes de enseñanza, siendo éstos la instrucción directa, el sistema personalizado, el aprendizaje cooperativo, la educación deportiva, la enseñanza entre iguales, el modelo de indagación, los juegos tácticos y el modelo de responsabilidad personal y social.

Respecto al concepto de «estilo o método», Manterola (2002) se refiriere a lo mismo con ambos términos, considerándolo como las diferentes modalidades que puede presentar un modelo, es decir, considerándolo parte del modelo de enseñanza.

Sin embargo, Delgado (1991) define el método como los caminos que permitirán que el alumno llegue a alcanzar los objetivos de enseñanza.

Otro concepto a conocer es el de intervención didáctica, el cual es definido por Zhang (2007) como un factor importante para llevar a cabo el proceso de enseñanza-aprendizaje, hablando también del término estilos de enseñanza, el cual describe las diferentes relaciones que se establecen entre el profesor y los alumnos.

Fecha recepción: 07-03-16. Fecha de aceptación: 30-06-16 María Fernández Rivas mespada@ucjc.edu
Mosston (1978) define los estilos de enseñanza como aquello que permiten mostrar cómo es la interacción entre el profesor y el alumno en la toma de decisiones del proceso de enseñanza-aprendizaje, permitiendo que ambos tengan su rol correspondiente dentro del proceso.

Más tarde, Delgado (1991) los define como la forma en que los elementos del proceso didáctico se relacionan, presentándose en el diseño instructivo y en cómo el profesor presenta e imparte la materia.

De este modo, existen dos autores que llevan a cabo propuestas importantes acerca de los estilos de enseñanza en Educación Física para facilitar la actuación del profesor en el aula y aportarle herramientas para llevar a cabo la enseñanza en función de las necesidades de los alumnos. Por un lado, Mosston (1978) propone el espectro de estilos de enseñanza basándose en la necesidad de clarificar el comportamiento del profesor. En este período (período de controversia, 1966-1986) se incluye un modelo que implica los diferentes canales de desarrollo del alumno (físico, cognitivo, emocional y social) (Mosston, 1978).

Este mismo autor, en un período de no controversia (1986act.), propone otra clasificación sustituyendo el término de canal de desarrollo por el de objetivo, siendo los estilos de enseñanza incluidos en esta clasificación el estilo de mando directo, enseñanza basada en la tarea, enseñanza recíproca, autoevaluación, estilos de inclusión, descubrimiento guiado, divergente, programa individualizado, para alumnos iniciados y autoenseñanza (Mosston \& Ashworth, 1993).

Por último, Delgado (1991), lleva a cabo una propuesta a partir de la de Mosston y Ashworth (1993), clasificando los estilos de enseñanza en familias según los objetivos a alcanzar: estilos tradicionales, estilos que posibilitan la participación del alumno, estilos que fomentan la individualización, que implican cognitivamente a los alumnos, que favorecen la socialización y que favorecen la creatividad. 
Además de los estilos de enseñanza vistos anteriormente, desde un punto de vista pedagógico más general existen otras propuestas de estilos de enseñanza, debido a que se consideran de gran importancia en el proceso de enseñanza-aprendizaje. Así vamos a presentar algunas de ellas por orden cronológico.

\begin{tabular}{|c|c|}
\hline Autor & Estilos de enseñanza \\
\hline Gordon (1959) & $\begin{array}{l}\text { Estilo instrumental } \\
\text { Estilo expresivo } \\
\text { Estilo instrumental y expresivo }\end{array}$ \\
\hline Flanders (1977) & $\begin{array}{l}\text { Estilo directo } \\
\text { Estilo indirecto }\end{array}$ \\
\hline Bennett (1979) & $\begin{array}{l}\text { Estilos de enseñanza progresistas o liberales } \\
\text { Estilos tradicionales o formales } \\
\text { Estilos mixtos }\end{array}$ \\
\hline Brostrom (1979) & $\begin{array}{l}\text { Estilo doctor } \\
\text { Estilo experto } \\
\text { Estilo entrenador } \\
\text { Estilo humanista }\end{array}$ \\
\hline Grasha (1996) & $\begin{array}{l}\text { Estilo del profesor experto } \\
\text { Estilo de autoridad formal } \\
\text { Estilo personal } \\
\text { Estilo facilitador } \\
\text { Estilo delegador }\end{array}$ \\
\hline
\end{tabular}

Cabe destacar que los estilos de enseñanza son utilizados para aportar conocimientos a los alumnos de una forma más eficaz, ya que se sugiere el uso de la motivación e interés (Shen \& Chen, 2007), lo que da lugar a un aprendizaje más significativo, y por lo tanto, más duradero (López \& Moreno, 2002).

Además, se llega a la conclusión, gracias a Biddle y Goudas (1993) y Delgado (1996), que el dominio y utilización de diferentes estilos de enseñanza ayudan al profesor de Educación Física, permitiendo realizar una buena planificación de las sesiones, dando lugar a un clima positivo en el aula y a un aumento de la motivación de los alumnos, y por lo tanto, una mejora del aprendizaje. Además, el profesor no solo debe utilizar todos los estilos de enseñanza, sino que tiene que ser capaz de combinarlos o crear estilos nuevos, sabiendo que no hay ningún estilo de enseñanza mejor que otro y que su aplicación depende de los alumnos y del contexto (González-Peiteado \& Aznar-Cuadrado, 2010; Mosston \& Ashworth, 1993; Sicilia \& Delgado, 2002).

Un buen profesor no solo debe utilizar metodologías coherentes, sino que debe disponer de ciertas habilidades docentes que posibiliten el buen aprendizaje de los alumnos (Ballesta, Izquierdo \& Romero, 2011).

Respecto a los estilos de enseñanza existen numerosas investigaciones a nivel internacional que pretenden profundizar y analizar acerca de cuál es su influencia y utilización en las clases de Educación Física.

En esta línea, investigaciones como las de Curtner-Smith et al. (2001), Jaakkola y Watt (2011) y Hewitt y Kenneth (2013), muestran que los estilos de enseñanza más aplicados a la hora de llevar a cabo las diferentes actividades en las clases de Educación Física son los estilos de mando directo y el estilo de práctica. Además, Cothran et al. (2005) afirman que los profesores de diferentes países siempre combinan varios estilos de enseñanza en sus clases de Educación Física.

Otras investigaciones muestran cuáles son los estilos de enseñanza más efectivos a la hora de desarrollar las diferentes capacidades en el alumno, observando que son estilos como el mando directo y la asignación de tareas los que más influyen tanto en el rendimiento como en la retención de contenidos de los alumnos (Boyce, 1992; Derri \& Pachta, 2007; Zeng, Leung, Liu \& Bian, 2009). Por otro lado, son los estilos de descubrimiento guiado, enseñanza recíproca, programas individuales y autoevaluación los que influyen principalmente en el ambiente de clase, a nivel afectivo y cognitivo, y en la motivación de los alumnos, y por lo tanto en su rendimiento académico (Morgan, et al., 2005; Salvara, et al., 2006; Patmanoglou, Mantis, Digelidis, Tsigilis \& Papapetrou, 2008; Kolovelonis, Goudas \& Gerodimos, 2011).

Por último, respecto a los estilos de enseñanza más aceptados, los profesores prefieren estilos de enseñanza como los estilos participativos, individuales, creativos, socializadores y cognitivos, rechazando los estilos de enseñanza tradicionales (Isaza \& Henao, 2012), mientras que el estilo de inclusión es el preferido por los alumnos (Sánchez, et al., 2012).

\section{Objetivos de la investigación}

- Analizar cómo es la formación inicial y la percepción sobre la aplicación de los estilos de enseñanza en Educación Física en función del sexo del profesorado.

- Describir cómo es la formación inicial y la percepción sobre la aplicación de los estilos de enseñanza en Educación Física en función de la edad del profesorado.

- Determinar cómo es la formación inicial y la percepción sobre la aplicación de los estilos de enseñanza en Educación Física en función de la titulación del profesorado.

- Conocer cuáles son los obstáculos encontrados a la hora de aplicar los estilos de enseñanza y la posibilidad de más investigaciones sobre el tema.

\section{Material y método}

Se lleva a cabo una investigación cuantitativa, descriptiva y no experimental, (Anguera, 1992; Cea D’Ancona, 2001; González Tirados, 2009), siendo las variables del estudio las siguientes:

\begin{tabular}{|c|c|c|}
\hline Variables dependientes & $\begin{array}{l}\text { Variables } \\
\text { independientes }\end{array}$ & Grupos \\
\hline \multirow{3}{*}{$\begin{array}{l}\text { La formación inicial y la percepción } \\
\text { sobre el uso de los estilos de } \\
\text { enseñanza en Educación Física }\end{array}$} & Sexo & $\begin{array}{l}\text { Hombres. } \\
\text { Mujeres }\end{array}$ \\
\hline & Edad & $\begin{array}{l}\text { Menores de } 30 \text { años. } \\
\text { Entre } 31 \text { y } 40 \text { años. } \\
\text { Entre } 41 \text { y } 50 . \\
\text { Mayores de } 51 \text { años. }\end{array}$ \\
\hline & Titulación & $\begin{array}{l}\text { Licenciados/ graduados en Ciencias } \\
\text { Actividad Física y Deporte } \\
\text { Diplomados/ graduados en Magisterio } \\
\text { de Educación Física. } \\
\text { Ambas titulaciones }\end{array}$ \\
\hline $\begin{array}{l}\text { Obstáculos encontrados a la hora } \\
\text { de poner en práctica los estilos de } \\
\text { enseñanza y posibilidad de más } \\
\text { investigaciones sobre el tema }\end{array}$ & Frecuencias & \\
\hline
\end{tabular}

\section{Participantes}

Para llevar a cabo la investigación, se ha utilizado una muestra de 455 profesores, de los cuales el 70,8\% de la muestra son hombres y el $29,2 \%$ son mujeres. Además, el $47,7 \%$ de la muestra son diplomados o graduados en magisterio de Educación Física, el 21,1\% licenciados o graduados en Ciencias de Actividad Física y Deporte y el 31,2\% tienen ambas titulaciones. Por otro lado, respecto a la edad, el 17,4\% de la muestra se encuentran en el rango de edad de menos de 30 años, el 51,4\% pertenece al rango de entre 31 y 40 años, un 19,8\% de los profesores tienen entre 41 y 50 años y el 11,4\% de la muestra pertenece a profesores con una edad de 51 años o más.

Se ha utilizado un diseño muestral probabilístico por conglomerados y estratificado, seleccionando primero los municipios, luego los centros y por último los profesores a encuestar en cada centro educativo, todo ello de forma aleatoria (Rodríguez Osuna, 2002). 
Así, el universo de la muestra consta de 1659 centros educativos de la Comunidad de Madrid, siendo calculado gracias a los listados de centros educativos de primaria y segundaria de la Comunidad de Madrid (Comunidad de Madrid, 2014) y del listado de municipios y población (Instituto de estadística de la Comunidad de Madrid, 2013).

Para determinar el tamaño de la muestra, se ha utilizado la fórmula para poblaciones finitas, explicada por diferentes autores (Cea D’Ancona, 2004; Sierra Bravo, 2001), siendo así «P» y «Q» iguales, teniendo el valor del 50\% cada uno de ellos, un nivel de confianza determinado entre -2 sigmas $y+2$ sigmas, siendo valores de una distribución normal y estando situada la probabilidad en el 95,5\%, y un margen de error de $+4,75$ para la muestra establecida. Una vez que se ha aplicado la fórmula, el tamaño de la muestra obtenido ha sido el de 455 unidades de la población.

\section{Instrumento}

Se ha utilizado la entrevista estructurada como instrumento para la recogida de datos (Lussier \& Kimball, 2008). El cuestionario utilizado, llamado cuestionario para el análisis de los estilos de enseñanza utilizados en Educación Física, ha sido elaborado y validado por Guedea (2010) con una fiabilidad de alfa de Cronbach $\alpha=.702$.

Dicho cuestionario está formado por 32 preguntas divididas en diferentes dimensiones:

- Dimensión I: Importancia de los estilos de enseñanza.

- Dimensión II: Conocimiento, preparación, formación inicial y permanente sobre estilos de enseñanza.

- Dimensión III: Dificultades con los estilos de enseñanza.

- Dimensión IV: Frecuencia de utilización de los estilos de enseñanza.

- Dimensión V: Grado de aceptación de los estilos de enseñanza.

- Dimensión VI: Aceptación de los alumnos en el medio ambiente físico, del contenido de la enseñanza, como factores que influyen en la utilización de los estilos de enseñanza.

En este caso, se han utilizado dos preguntas de la dimensión II, las cuatro incluidas dentro de la dimensión III, y dos preguntas de la dimensión VI.

\section{Procedimiento}

Este estudio tuvo lugar en un momento determinado del año, que abarca todo el curso académico 2014- 2015, a lo que se le denomina estudio transversal (Sierra Bravo, 2001), llevado a cabo por una única persona, lo cual favorece la eficacia de la recogida de datos.

Para el análisis de los datos, se ha realizado un análisis descriptivo a través de tablas de frecuencias y un análisis inferencial a través de diferentes pruebas (t-Student, ANOVA y Welch) según las variables sean dicotómicas (sexo) o politómicas (edad y titulación del profesorado) utilizando el programa estadístico para el análisis de datos SPSS ${ }^{\circledR}$, Versión 20.

\section{Resultados}

En primer lugar, se lleva a cabo el análisis inferencial analizando las preguntas en función del sexo, para lo que se utiliza la prueba t-Student (tabla 3), observando que en la prueba de Levene para la igualdad de varianzas, se asume igualdad de varianzas ( $p>$.05) en todos los ítems, donde al acudir a la prueba t, no se observan diferencias significativas entre las variables $(p>.05)$.

\begin{tabular}{|c|c|c|c|c|c|c|}
\hline Ítems & & F & Sig. & $\mathrm{T}$ & gl & $\begin{array}{c}\text { Sig. } \\
\text { (bilateral) }\end{array}$ \\
\hline \multirow{2}{*}{$\begin{array}{l}\text { 1. ¿Cómo fue la formación } \\
\text { práctica sobre EE? }\end{array}$} & $\begin{array}{l}\text { Se han asumido } \\
\text { varianzas iguales }\end{array}$ & 0,009 & 0,926 & 1,620 & 453 & 106 \\
\hline & $\begin{array}{l}\text { No se han asumido } \\
\text { varianzas iguales }\end{array}$ & & & 1,600 & 239,530 & 111 \\
\hline \multirow{2}{*}{$\begin{array}{l}\text { 2. ¿Te sientes preparado para } \\
\text { usar los EE? }\end{array}$} & $\begin{array}{l}\text { Se han asumido } \\
\text { varianzas iguales }\end{array}$ & 0,112 & 0,739 & 1,600 & 453 & 110 \\
\hline & $\begin{array}{l}\text { No se han asumido } \\
\text { varianzas iguales }\end{array}$ & & & 1,544 & 228,694 & 124 \\
\hline \multirow{2}{*}{$\begin{array}{l}\text { 3. ¿Cómo crees que te has } \\
\text { desempeñado con los estilos de } \\
\text { enseñanza? }\end{array}$} & $\begin{array}{l}\text { Se han asumido } \\
\text { varianzas iguales }\end{array}$ & 0,57 & 0,451 & 0,022 & 453 & 0,983 \\
\hline & $\begin{array}{l}\text { No se han asumido } \\
\text { varianzas iguales }\end{array}$ & & & 0,021 & 225,109 & 0,984 \\
\hline \multirow{2}{*}{$\begin{array}{l}\text { 4. ¿Consideras difícil el uso de } \\
\text { los estilos de enseñanza? }\end{array}$} & $\begin{array}{l}\text { Se han asumido } \\
\text { varianzas iguales }\end{array}$ & 2,501 & 0,114 & $-0,614$ & 453 & 0,54 \\
\hline & $\begin{array}{l}\text { No se han asumido } \\
\text { varianzas iguales }\end{array}$ & & & $-0,592$ & 228,054 & 0,555 \\
\hline \multirow{2}{*}{$\begin{array}{l}\text { 5. ¿Los estilos de enseñanza } \\
\text { ayudan en el desarrollo de la } \\
\text { clase? }\end{array}$} & $\begin{array}{l}\text { Se han asumido } \\
\text { varianzas iguales }\end{array}$ & 3,821 & 0,051 & 1,086 & 453 & 0,278 \\
\hline & $\begin{array}{l}\text { No se han asumido } \\
\text { varianzas iguales }\end{array}$ & & & 1,031 & 221,479 & 0,303 \\
\hline \multirow{2}{*}{$\begin{array}{l}\text { 6. ¿Qué grado de satisfacción } \\
\text { han aportado los estilos de } \\
\text { enseñanza? }\end{array}$} & $\begin{array}{l}\text { Se han asumido } \\
\text { varianzas iguales }\end{array}$ & 1,532 & 0,216 & 0,239 & 453 & 0,811 \\
\hline & $\begin{array}{l}\text { No se han asumido } \\
\text { varianzas iguales }\end{array}$ & & & 0,235 & 236,96 & 0,815 \\
\hline
\end{tabular}

En segundo lugar, se analizan los ítems en función de la edad. Para ello, se lleva a cabo la prueba de homogeneidad de Levene, donde se observa que únicamente en dos de los seis ítems existen diferencias significativas $(p<.05)$, los cuales son el ítem 4, sobre si consideran difícil la aplicación de los estilos de enseñanza $(p=.04)$, y el ítem 6 acerca de si la aplicación de los estilos de enseñanza ayuda en el desarrollo de las sesiones $(p=.006)$.

Los ítems en los que se apreciaron diferencias significativas a través de la prueba de homogeneidad de Levene son sometidos a la prueba de Welch, donde solo se vieron diferencias entre las variables en el ítem 4 sobre la dificultad de la aplicación de los estilos de enseñanza $(p=.000)$.

Tras estos resultados, se realiza la prueba post-hoc de Games-Howell (tabla 4) en el ítem 4 para conocer entre qué grupos se encuentran dichas diferencias, mostrando que se encuentran entre los profesores de entre 31 y 50 años (31-40, $p=.000$; 41-50, $p=.039$ ) y los profesores menores de 30 años, siendo los profesores de entre 31 y 50 años los que consideran más difícil la aplicación de los estilos de enseñanza frente a los profesores más jóvenes (menores de 30 años), quienes no lo consideran difícil.

\begin{tabular}{|c|c|c|c|c|c|}
\hline Ítems & (I) Edad & (J) Edad & $\begin{array}{c}\text { Diferencia de } \\
\text { medias (I-J) }\end{array}$ & Error típico & Sig. \\
\hline \multirow{12}{*}{$\begin{array}{l}\text { 2. ¿Consideras difícil el } \\
\text { uso de los estilos de } \\
\text { enseñanza? }\end{array}$} & \multirow[t]{3}{*}{$\begin{array}{c}30 \text { o } \\
\text { menos }\end{array}$} & $31-40$ & $-1,194^{*}$ & 0,273 & 0 \\
\hline & & $41-50$ & $\begin{array}{l}-903^{*} \\
\end{array}$ & 0,335 & 0,039 \\
\hline & & 51 o más & $-0,82$ & 0,417 & 0,209 \\
\hline & \multirow[t]{3}{*}{$31-40$} & 30 o menos & $1,194^{*}$ & 0,273 & 0 \\
\hline & & $41-50$ & 0,291 & 0,269 & 0,701 \\
\hline & & 51 o más & 0,374 & 0,366 & 0,738 \\
\hline & \multirow[t]{3}{*}{$41-50$} & 30 o menos & ,903* & 0,335 & 0,039 \\
\hline & & $31-40$ & $-0,291$ & 0,269 & 0,701 \\
\hline & & 51 o más & 0,083 & 0,414 & 0,997 \\
\hline & \multirow[t]{3}{*}{51 o más } & 30 o menos & 0,82 & 0,417 & 0,209 \\
\hline & & $31-40$ & $-0,374$ & 0,366 & 0,738 \\
\hline & & $41-50$ & $-0,083$ & 0,414 & 0,997 \\
\hline
\end{tabular}

Por otro lado, los ítems en los que no se observaron diferencias en la prueba de homogeneidad de Levene ( $\mathrm{p}>.05$ ) son sometidos al test ANOVA, siendo estos ítems el 1 sobre cómo fue la formación práctica de los profesores de Educación Física, el ítem 2 sobre si éstos se sienten preparados para usarlos, ítem 3 sobre cómo creen que se han desempeñado a la hora de ponerlos en práctica y el ítem 6 sobre el grado de satisfacción personal en el momento de aplicar los estilos de enseñanza. Así, fueron dos los ítems donde se ven diferencias significativas entre las varia- 
bles, el ítem $3(p=.005)$ y el $6(p=.042)$.

Después, se recurre a la prueba post-hoc Tukey (tabla 5) para conocer entre qué grupo se encuentran dichas diferencias, observando que en el ítem 3 , acerca de cómo los docentes creen que se han desempeñado a la hora aplicar los estilos de enseñanza, son los docentes con edades menores de 40 años (profesores menores de 30, $p=.02 ; 31-40, p=.014$ ) los que consideran que se han desempeñado mejor que aquellos profesores mayores de 51 años.

En el ítem 6, no se observan diferencias significativas entre los diferentes grupos de edad ( $p>.05)$.

\begin{tabular}{|c|c|c|c|c|c|}
\hline Ítems & (I) Edad & (J) Edad & $\begin{array}{c}\text { Diferencia de } \\
\text { medias (I-J) }\end{array}$ & $\begin{array}{l}\text { Error } \\
\text { típico }\end{array}$ & Sig. \\
\hline \multirow{12}{*}{$\begin{array}{l}\text { 1. ¿Cómo crees que te has } \\
\text { desempeñado con los estilos de } \\
\text { enseñanza? }\end{array}$} & \multirow[t]{3}{*}{30 o menos } & $31-40$ & 0,066 & 0,161 & 0,977 \\
\hline & & $41-50$ & 0,373 & 0,191 & 0,208 \\
\hline & & 51 o más & ,641* & 0,221 & 0,02 \\
\hline & \multirow[t]{3}{*}{$31-40$} & 30 o menos & $-0,066$ & 0,161 & 0,977 \\
\hline & & 41-50 & 0,307 & 0,154 & 0,19 \\
\hline & & 51 o más & ,575* & 0,19 & 0,014 \\
\hline & \multirow[t]{3}{*}{$41-50$} & 30 o menos & $-0,373$ & 0,191 & 0,208 \\
\hline & & $31-40$ & $-0,307$ & 0,154 & 0,19 \\
\hline & & 51 o más & 0,268 & 0,216 & 0,6 \\
\hline & \multirow[t]{3}{*}{51 o más } & 30 o menos &,$- 641 *$ & 0,221 & 0,02 \\
\hline & & $31-40$ &,$- 575^{*}$ & 0,19 & 0,014 \\
\hline & & $41-50$ & $-0,268$ & 0,216 & 0,6 \\
\hline \multirow{12}{*}{$\begin{array}{l}\text { 4. ¿Qué grado de satisfacción han } \\
\text { aportado los estilos de enseñanza? }\end{array}$} & \multirow[t]{3}{*}{30 o menos } & $31-40$ & 0,178 & 0,108 & 0,354 \\
\hline & & $41-50$ & 0,327 & 0,128 & 0,054 \\
\hline & & 51 o más & 0,338 & 0,148 & 0,104 \\
\hline & \multirow[t]{3}{*}{$31-40$} & 30 o menos & $-0,178$ & 0,108 & 0,354 \\
\hline & & $41-50$ & 0,149 & 0,103 & 0,473 \\
\hline & & 51 o más & 0,16 & 0,127 & 0,59 \\
\hline & \multirow[t]{3}{*}{$41-50$} & 30 o menos & $-0,327$ & 0,128 & 0,054 \\
\hline & & $31-40$ & $-0,149$ & 0,103 & 0,473 \\
\hline & & 51 o más & 0,012 & 0,145 & 1 \\
\hline & \multirow[t]{3}{*}{51 o más } & 30 o menos & $-0,338$ & 0,148 & 0,104 \\
\hline & & $31-40$ & $-0,16$ & 0,127 & 0,59 \\
\hline & & $41-50$ & $-0,012$ & 0,145 & 1 \\
\hline
\end{tabular}

En tercer lugar, se analizan los diferentes ítems según la titulación de los profesores. Partiendo de la prueba de Levene, se observa que en el ítem 3 hay diferencias de varianza acerca de si los estilos de enseñanza ayudan a desarrollar mentalmente las sesiones de Educación Física $(p<.05)$.

En este ítem donde se observaron diferencias de varianzas, para ver si existen diferencias significativas entre las variables, se recurre a la prueba de Welch, donde no se observa ninguna diferencia entre ellas ( $p>05)$.

Después, se analizan los ítems donde no se observaron diferencias de varianza ( $p>$.05) utilizando el test ANOVA, donde se observan únicamente diferencias significativas en el ítem 1 acerca de cómo fue la formación práctica y la titulación ( $p=.002$ ).

Para conocer entre que grupos de titulación se encuentran estas diferencias, se lleva a cabo la prueba post-hoc Tukey (tabla 6), donde se observan diferencias entre diplomados o graduados en magisterio de Educación Física respecto a los licenciados o graduados en CAFYD y profesores con ambas titulaciones, siendo tanto los licenciados o graduados en CAFYD $(p=.008)$ como los profesores con ambas titulaciones $(p=.01)$ los que consideran que su formación práctica fue buena, al contrario que los diplomados o graduados en magisterio de Educación Física.

Por último, se lleva a cabo el análisis descriptivo a través de frecuencias de los dos últimos ítems, el ítem 7 acerca de cuáles son los obstáculos más importantes a la hora de llevar poner en práctica los estilos de enseñanza en las clases de Educación Física, y el ítem 8 sobre si los profesores sugieren hacer investigación sobre los estilos de enseñanza.

Respecto al ítem 7 sobre los obstáculos encontrados a la hora de aplicar los estilos de enseñanza, las respuestas obtenidas fueron la falta de materiales, espacios, ratio de alumnos elevado, características y actitud de los alumnos ante la asigna-
Tabla 6

Prueba post-hoc de Tukey respecto a la variable de titulación.

\begin{tabular}{|c|c|c|c|c|c|}
\hline Ítems & (I) Titulación inicial & (J) Titulación inicial & $\begin{array}{c}\text { Diferencia de } \\
\text { medias (I-J) }\end{array}$ & $\begin{array}{l}\text { Error } \\
\text { típico } \\
\end{array}$ & Sig. \\
\hline \multirow{6}{*}{$\begin{array}{l}\text { 1. ¿Cómo fue } \\
\text { la formación } \\
\text { práctica sobre } \\
\text { estilos de } \\
\text { enseñanza? }\end{array}$} & \multirow[t]{2}{*}{ Diplomado/Grado Magisterio } & $\begin{array}{l}\text { Licenciado/ Grado } \\
\text { CAFYD }\end{array}$ &,$- 399 *$ & 0,133 & 0,008 \\
\hline & & $\begin{array}{c}\text { Diplomado y } \\
\text { Licenciado }\end{array}$ &,$- 343^{*}$ & 0,118 & 0,01 \\
\hline & \multirow{2}{*}{ Licenciado/ Grado CAFYD } & $\begin{array}{c}\text { Diplomado/Grado } \\
\text { Magisterio }\end{array}$ & ,399* & 0,133 & 0,008 \\
\hline & & $\begin{array}{c}\text { Diplomado y } \\
\text { Licenciado }\end{array}$ & 0,056 & 0,144 & 0,92 \\
\hline & \multirow[t]{2}{*}{ Diplomado y Licenciado } & $\begin{array}{c}\text { Diplomado/Grado } \\
\text { Magisterio }\end{array}$ &, $343^{*}$ & 0,118 & 0,01 \\
\hline & & $\begin{array}{l}\text { Licenciado/ Grado } \\
\text { CAFYD }\end{array}$ & $-0,056$ & 0,144 & 0,92 \\
\hline
\end{tabular}

* La diferencia de medias es significativa al nivel 0.05 .

tura, falta de tiempo en las clases, necesidad de planificar, el bilingüismo, la falta de formación inicial del profesorado, cambio continuo de la legislación de educación o ningún obstáculo.

Así, de entre todas las respuestas obtenidas, el obstáculo más importante a la hora de llevarlos a la práctica son las características y actitud de los alumnos (27,7\%). En muchos casos, se ha observado que los profesores no encuentran ningún obstáculo para aplicar los estilos de enseñanza en sus clases (22\%). El resto de respuestas obtenidas no superan el $10 \%$ de la muestra (tabla 7).

Tabla 7.

Frecuencias sobre cuáles son los obstáculos más importantes a la hora de poner en práctica los estilos de enseñanza.

Respuestas

Materiales

Espacios

Ratio elevado

Características y actitud de los alumnos

Falta de tiempo

Necesidad de planificar

Espacios, materiales y ratio

Ratio elevado y tiempo rio

Bilingüismo

Formación inicial insuficiente sobre estilos de enseñanza

Formació

Material, espacio y características del grupo

Ratio elevado y espacio

Ratio elevado y características de los alumnos

Espacio, material y tiempo

Ratio elevado, tiempo reducido y características de los alumnos

Cambio continuo de legislación

Espacios y materiales

Tiempo reducido y características y actitud de alumnos

Total

Respecto al ítem 8 acerca de si los profesores consideran necesaria más investigación sobre los estilos de enseñanza en Educación Física, se ha obtenido que la mayoría de los profesores consideran que es bueno seguir investigando para la mejora de la educación (59,3\%). A pesar de ello, hay profesores que consideran que no es necesario llevar a cabo más investigaciones sobre estilos de enseñanza en Educación Física ya que es suficiente con los estilos de enseñanza que existen (18\%) y hay que conocerlos mejor antes de seguir investigando (14,9\%). El resto de respuestas obtenidas no supera el 6\% de la muestra (tabla 8).

Tabla 8.

Frecuencias sobre si los profesores sugieren hacer investigaciones sobre más estilos de enseñanza específicos para Educación Física.

\begin{tabular}{lcc}
\hline Respuestas & Frecuencia & Porcentaje \\
\hline Si, para mejorar en la educación & 270 & 59,3 \\
No, hay que conocer mejor lo que ya existe & 68 & 14,9 \\
Si, nunca hay que dejar de investigar & 27 & 5,9 \\
No, es suficiente con lo que hay & 82 & 18 \\
No, no son prácticos & 2 & 0,4 \\
Sí, porque alumnos y educación van cambiando & 6 & 1,3 \\
\hline Total & 455 & 100 \\
\hline
\end{tabular}

\section{Discusión}

En primer lugar, respecto al sexo, no se observan diferencias significativas ni en la formación inicial y permanente del profesorado ni en las sensaciones percibidas por los profesores a la hora de aplicar los estilos de enseñanza en el aula de Educación Física. Sin embargo, en la investigación realizada por Del- 
gado y Zurita (2003), los autores afirma que los hombres son quienes valoran más las diferentes teorías de la Educación Física, teniendo una concepción de la materia como recreación, mientras que las mujeres la valoran más como materia relacionada con la salud. Además, Abad, Giménez, Robles y Rodríguez (2011) afirman que los entrenadores de fútbol, en su mayoría hombres, utilizan metodologías directivas. A pesar de ello, Méndez-Giménez, Fernández-Río y Méndez-Alonso (2015) afirman que un modelo de educación deportiva es mucho más motivador que los métodos tradicionales, favoreciendo la actitud positiva de los alumnos hacia las tareas.

En segundo lugar, respecto a la edad, se observan diferencias significativas acerca de las sensaciones de los profesores a la hora de poner en práctica los diferentes estilos de enseñanza, encontrándose éstas entre los profesores más jóvenes, menores de 40 años, y los mayores de 51 años, siendo los menores de 40 años quienes perciben que se han desempeñado mejor a la hora de aplicar los estilos de enseñanza en comparación con los profesores más veteranos. Además, los profesores menores de 30 años son los que consideran que es más fácil la aplicación de los estilos de enseñanza respecto a los profesores con edades comprendidas entre 31 y 50 años. En este sentido, la experiencia docente, como afirman los estudios de Sicilia y Fernández (2006) y Vera (2010), tiene gran importancia a la hora de llevar a cabo las diferentes actividades, sin embargo, también es necesaria la formación permanente para poder adaptar su metodología a las características sociales del momento para una mejor educación, lo que les aportaría una mayor facilidad a la hora de aplicar los estilos de enseñanza (Martínez \& Díaz, 2003; Pastor, 2008; Romero, 2008; Salina, 2007; Zabala, 2003). Por otro lado, Abdeddaim, Sebbane, Zitouni, Zubiaur y Boumesdji (2016) también afirman que un período prolongado de una actividad, mayor a 10 años de experiencia, permite hacer al profesor experto en dicha actividad. Además, para evitar los problemas de organización en las clases de Educación Física, se utiliza la instrucción directa como técnica de enseñanza, facilitando la puesta en práctica de las diferentes metodologías de enseñanza (SáenzLópez, 2000).

En tercer lugar, respecto a la variable de titulación, se observan diferencias significativas acerca de cómo fue su formación práctica sobre estilos de enseñanza, los profesores licenciados o graduados en Ciencias de la Actividad Física y el Deporte y profesores con dos titulaciones son los que consideran que fue buena en comparación con las respuestas dadas por los diplomados o graduados en magisterio de Educación Física. Cabe destacar que López (2012) en su investigación afirma que la mayoría de los profesores de educación secundaria, quienes tienen la titulación de licenciados o graduados en Ciencias de Actividad Física y Deporte, que tienen una experiencia docente entre siete y veinticuatro años, afirman realizar cursos de reciclaje periódicamente. También, Soriano y Delgado (2011) afirman que la formación continua es un elemento imprescindible para llegar a ser un profesor excelente, mientras que la formación inicial únicamente es el comienzo de la profesión de docente. Así, hay numerosas investigaciones, tales como las de Martínez y Díaz (2003), Romero (2008), Salina (2007) y Zabala (2003), que afirman que la formación práctica, la cual corresponde al prácticum de la formación universitaria elegida, tiene unos objetivos enfocados tanto a la formación de conocimientos como a la adaptación de estos conocimientos al mundo laboral. Por ello, existe la necesidad de atender a los cambios que se producen en la sociedad y en la educación a la hora de impartir la formación universitaria y permanente a los profesores, para así poder llevar a cabo una enseñanza adecuada a la situación social y educativa del momento en el aula, teniendo la capacidad de utilizar y adaptar los conocimientos teóricos a la práctica (Pastor, 2008). Sin embargo, hay investigaciones que afirman que para ciertos contenidos más innovadores en Educación Física, como es la expresión corporal, los profesores no dedican tiempo suficiente a la programación, actualización y preparación de las actividades, en concreto los profesores de primaria (Montávez, 2011).

Por último, se observa que uno de los obstáculos más importantes que hacen difícil a los profesores la aplicación de los estilos de enseñanza son las características de los alumnos y sus actitudes, ya que los grupos formados son heterogéneos y es necesaria la adaptación de los contenidos a las necesidades de los alumnos. En esta línea, las investigaciones de Alemany y Villuendas (2004), Molina y Marqués (2009) y Sola (1997), afirman que para poder llevar a cabo adaptaciones en el contenido del programa y así atender las diferencias individuales de los alumnos, es necesaria una buena formación tanto inicial como permanente. Por esto, no es suficiente la experiencia docente, sino que es necesaria la formación permanente (Sicilia y Fernández, 2006; Vera, 2010). Por otro lado, Pankratius (1997) en su investigación aborda la incertidumbre que tiene lugar cuando no se lleva a cabo una formación permanente, ya que faltan recursos para adaptar los contenidos, lo que da lugar al cansancio de los profesores y por lo tanto surgen los posibles obstáculos. Sin embargo, otros profesores, independientemente de la heterogeneidad del grupo, no encuentran ningún obstáculo que dificulte la aplicación de los estilos de enseñanza de Educación Física.

Además, se considera necesaria la actualización de los estilos de enseñanza ya que toda sociedad está en continuo cambio, afirmando los profesores de Educación Física que es conveniente más investigación sobre el tema para mejorar en la educación y adaptar los contenidos a las necesidades de los alumnos. Así lo afirman algunos autores en sus investigaciones, ya que para la mejora de la educación, es necesario adaptar las metodologías a las características de los alumnos y por lo tanto a las características sociales del momento (Martínez \& Díaz, 2003; Pastor, 2008; Salina, 2007; Zabala, 2003). Además, Soriano y Delgado (2011) afirman que es necesario profundizar en las competencias profesionales con el objetivo de asegurar la buena enseñanza de Educación Física. Sin embargo, una minoría de profesores de Educación Física, considera que los estilos de enseñanza existentes son suficientes, siendo necesario conocerlos en profundidad antes de seguir investigando, para lo que sería necesaria la formación permanente del profesorado (Sicilia \& Fernández, 2006).

\section{Conclusiones}

Los únicos que consideran haber tenido una buena formación práctica acerca de los estilos de enseñanza en Educación Física son los profesores licenciados o graduados en Ciencias de Actividad Física y Deporte y profesores que además de esa titulación tienen la de diplomado o graduado en magisterio de Educación Física .

Además, a pesar de ser los profesores menores de 40 años los que consideran que se han desempeñado bien a la hora de aplicar los estilos de enseñanza frente a los mayores de 51 años, son los profesores menores de 30 a los que les resulta más fácil utilizarlos en las clases de Educación Física respecto a los pro- 
fesores con edades comprendidas entre 31 y 50 años.

Pueden existir obstáculos a la hora de poner en práctica los estilos de enseñanza. Así, la mayoría de los profesores consideran que las características de los alumnos y sus actitudes ante la Educación Física pueden dificultar su utilización.

Algunos profesores de Educación Física consideran que es necesario seguir investigando para mejorar en la educación.

Los resultados obtenidos aportan una información importante respecto al conocimiento y puesta en práctica de metodologías de enseñanza como son los estilos de enseñanza. Así, se muestra cómo es la formación inicial del profesorado de Educación Física con respecto a los estilos de enseñanza, siendo posible su mejora para la posterior puesta en práctica de metodologías por parte de los profesores, intentando eliminar los posibles obstáculos presentados en el aula, siendo necesario profundizar aún más en el tema de estudio.

\section{Referencias}

Abad, M.T., Giménez, F.J., Robles, J., \& Rodríguez, J.M. (2011). Perfil, experiencia y métodos de enseñanza de los entrenadores de jóvenes futbolistas en la provincia de Huelva. Retos. Nuevas tendencias en Educación Física, Deporte y Recreación. 20, 21-25.

Abdeddaim, A., Sebbane, M., Zitouni, A., Zubiaur, M., \& Boumesdji, A. (2016). Efecto de la base de conocimientos y de la memoria experta en una tarea de detección de errores de arbitraje en balonmano. Revista Iberoamericana de Psicología del ejercicio y el deporte, 11(2), 239-245.

Alemany, I., \& Villuendas, M. D. (2004). Las Actitudes del Profesorado hacia el Alumnado con Necesidades Educativas Especiales. Convergencia, (34), 183-215.

Anguera, M. T. (1992). Metodología de la observación en las ciencias humanas. Madrid: Catedra.

Ballesta, F.; Izquierdo, T., \& Romero, B. (2011). Percepción del alumnado de Pedagogía ante el uso de metodologías activas. Educación Siglo XXI, 2(29), 353-368.

Bennet, S. N. (1979). Estilos de enseñanza y progreso de los alumnos. En García, D. M., Joaquín, M., Torres, P., y Vázquez, I. R., Estilos de enseñanza y las nuevas tecnologías en la educación. ReiDoCrea, 2, 219-225.

Biddle, S., \& Goudas, M. (1993). Reaching Styles, class climate and motivational in Physical Education. Journal Physical Education, Recreation and Dance, 3(24), 38-39.

Boyce, B. (1992). The effects of three styles of teaching on university students motor performance. Journal of Teaching in Physical Education, 11(4), 389- 401.

Brostrom, R. (1979) Training Style Inventory (TSI). En Jones, J.E. y Pfeiffer, J.W. (ed.). The 1979 annual handbook for group facilitators. Bories in human relations training. California: Editorial Jones and Pfeiffer.

Comunidad de Madrid (2014). Guía de centros docentes de la Comunidad de Madrid. En web: www.madrid.org/ centros_docentes/guia/index.html

Cea D’Ancona, M. Á. (2001). Metodología Cuantitativa. Estrategias y técnicas de investigación social. (3rd ed.). Madrid: Síntesis.

Cea D’Ancona, M. Á. (2004). Métodos de la encuesta. Teoría y práctica, errores y mejora. Madrid: Síntesis.

Cothran, D. J., Kulinna, P. H., Banville, D., Choi, E., AmadeEscot, C., MacPhail, A., \& Kirk, D. (2005). A cross- cultural investigation of the use of teaching styles. Research Quarterly for Exercise and Sport, 76(2), 193- 201.

Delgado, M. Á. (1991). Los estilos de enseñanza en la educación. Propuesta para una reforma de la enseñanza. Granada: I.C.E. Universidad de Granada.
Delgado, M. Á. (1996). Aplicaciones de los Estilos de Enseñanza en la Educación Primaria. En C. Romero (comps.), Estrategias Metodológicas para el Aprendizaje de los Contenidos de Educación Física Escolar (pp. 73-86). Granada: Promeco.

Delgado, M.Á., \& Zurita, F. (2003). Estudio de las teorías implícitas de la Educación Física en la formación inicial de los maestros en las diferentes especialidades de la facultad de ciencias de la educación de la universidad de Granada. Diferencias en función del género. Retos. Nuevas tendencias en Educación Física, Deporte y Recreación, 5, 27-38.

Derri, V., \& Pachta, M. (2007). Motor skills and concepts acquisition and retention: a comparison between two styles of teaching. International Journal of Sport Science, 63(1), $37-47$.

Flanders, N. (1977). Análisis de la interacción didáctica. En García, D. M., Joaquín, M., Torres, P., y Vázquez, I. R., Estilos de enseñanza y las nuevas tecnologías en la educación. ReiDoCrea, 2, 219- 225.

González-Peiteado, M., \& Aznar-Cuadrado, V. (2010). Aproximación al perfil de estilos de enseñanza en la formación inicial del profesorado de Educación Secundaria: nuevos canales, nuevos retos. Actas del I Congreso Internacional Reinventar la profesión docente, Málaga.

González Tirados, R. M. (2009). Documentos para la docencia. Bases conceptuales en el proceso de la investigación. Madrid: ICE Universidad Politécnica de Madrid.

Gordon, C. W. (1959). Die Schulklasse als soziales System. En García, D. M., Joaquín, M., Torres, P., y Vázquez, I. R., Estilos de enseñanza y las nuevas tecnologías en la educación. ReiDoCrea, 2, 219- 225.

Grasha, A. (1996). Teaching with style. A practical guide to enhancing learning by understanding teaching and learning styles. Cincinnati (EUA): Alliance.

Guedea, J. C. (2010). Análisis de los Estilos de Enseñanza utilizados por los profesores de Educación Física del nivel primaria en la ciudad Chihuahua. Universidad de Granada, Granada, España.

Hewitt, M., \& Kenneth, E. (2013). Estilos de enseñanza observados entre los entrenadores de juveniles y profesionales de Tenis Australia. ITF Coaching and Sport Science Review, 59(21), 6- 8.

Instituto de Estadística de la Comunidad de Madrid (2013). Demografía y población. En Web: http://www.madrid.org/ iestadis

Isaza, L., \& Henao, G. C. (2012). Actitudes- Estilos de enseñanza: su relación con el rendimiento académico. International Journal of Physical Education, 5(1), 133- 141.

Jaakkola, T., \& Watt, A. (2011). Finnish physical education teachers' self-reported use and perceptions of Mosston and Ashworth's teaching styles. Journal of Teaching in Physical Education, 30, 248- 262.

Kolovelonis, A.; Goudas, M., \& Gerodimos, V. (2011). The effects of the reciprocal and the self-check styles on pupils' performance in primary physical education. European Physical Education Review, 17(1), 35- 50.

López, A., \& Moreno, J. A. (2002). Aprendizaje de hechos y conceptos en educación física. Una propuesta metodológica. Apunts. Educación Física Y Deportes, 63, 16-26.

López, J.A. (2012). Calidad total de la materia de Educación Física: Adaptación del Modelo Europeo de Excelencia. El caso de los centros de Enseñanza Secundaria Obligatoria en la ciudad de Granada y su provincia. (Tesis Doctoral). Universidad de Granada, Granada.

Lussier, R. N., \& Kimball, D. C. (2008). Applied sport management skills. Unit States: Human Kinetics. 
Manterola, C., \& Otzen, T. (2014). Estudios observacionales. Los diseños utilizados con mayor frecuencia en investigación clínica. International Journal of Morphology, 32(2), 634-645.

Martínez, L., \& Díaz, B. (2003). El prácticum de Educación Física y la Iniciación de temas de reflexión en la construcción de la identidad profesional. In Actas del VII Symposium Internacional sobre el Prácticum (pp. 801-808). Pontevedra: Servicio de edición de la Universidad.

Méndez-Giménez, A., Fernández-Río, J. \& Méndez-Alonso, D. (2015). Modelo de educación deportiva versus modelo tradicional: efectos en la motivación y deportividad. Revista internacional de Medicina y Ciencias de la Actividad Física y del Deporte, 15(59), 449-466.

Metzler, M. (2005). Instructional models for physical education (2nd Ed.). Scottsdale, AZ: Holcomb Hathaway.

Molina, J., \& Marqués, C. (2009). Experiencia docente en Educación Física y alumnos con necesidades educativas especiales: estudio de correlación. Retos. Nuevas Tendencias En Educación Física, Deporte Y Recreación, (16), 16-21.

Montávez, M. (2011). La expresión corporal en la realidad educativa. Descripción y análisis de su enseñanza como punto de referencia para la mejora de la calidad docente en los centros públicos de la educación primaria de la ciudad de Córdoba. (Tesis doctoral). Universidad de Córdoba, Córdoba.

Morgan, K., Kingston, K., \& Sproule, J. (2005). Effects of different teaching styles on the teacher behaviours that influence motivational climate and pupils' motivation in physical education. European Physical Education Review, 11(3), 257- 285.

Mosston, M. (1978). La enseñanza de la Educación Física. Del comando al descubrimiento. Buenos Aires: Paidos.

Mosston, M., \& Ashworth, S. (1993). La enseñanza de la Educación Física. La reforma de los estilos de enseñanza. Barcelona: Hispano Europea.

Pankratius, W. (1997). Preservice teachers construct a view on teaching and learning styles. Action in Teacher Education, 18(4), 68- 76.

Pastor, J. L. (2008). La identidad como elemento común del espacio profesional y el ámbito académico. Revista Fuentes, 8, 36-52.

Patmanoglou, S., Mantis, K., Digelidis, N., Tsigilis, N., \& Papapetrou, L. (2008). The command and self- check styles for more effective teaching of tennis at the elementary school. International Journal of Physical Education, 45(1), 26- 32.

Rodríguez Osuna, J. (2002). La muestra: teoría y aplicación. En F. Alvira, M. García Ferrando y J. Ibáñez (Comps.), El análisis de la realidad social. Métodos y técnicas de investigación (3a ed.). Madrid: Alianza Editorial.
Romero, C. (2008). La dimensión personal de los sujetos participantes en su formación como futuros maestros en Educación Física a través del prácticum. Revista Fuentes, 8, 125142.

Sáenz-López, P. (2000). El maestro principiante de educación física. Publicaciones de la Universidad de Huelva.

Salina, D. (2007). EEES y Prácticum: ¿cómo encajar el prácticum en el nuevo marco?, en A. Cid et al (coords.). In Actas del IX Symposium Internacional sobre Prácticum y Prácticas en Empresas en la formación universitaria. (pp. 22-33). Universidad de Santiago, Vigo y la Coruña.

Salvara, M., Jess, M., Abbott, A., \& Bognár, J. (2006). A preliminary study to investigate the influence of different teaching styles on pupils' goal orientations in physical education. European Physical Education Review, 12(1), 5174

Sánchez, B., Byra, M., \& Wallhead, T. L. (2012). Students' perceptions of the command, practice, and inclusion styles of teaching. Physical Education and Sport Pedagogy, 17(3), 317- 330 .

Shen, B., \& Chen, A. (2007). An examination of learning profiles in physical education. Journal of Teaching in Physical Education, 2(26), 145-160.

Sicilia, Á, \& Delgado, M. Á. (2002). Educación Física y Estilos de Enseñanza. Madrid: INDE.

Sicilia, Á, \& Fernández, J. M. (2006). Ethics, politics and biopedagogy in physical education teacher education: easing the tension between the self and the group. Sport, Education and Society, 11(1), 1-20.

Sierra Bravo, R. (2001). Técnicas de investigación social. Teoría y ejercicios. Madrid: Thomson.

Sola, T. (1997). La formación inicial y su incidencia en la educación especial. En Sánchez, A. Y Torres.

Soriano, M.A., \& Delgado, M.A. (2011) Buenas Prácticas Profesionales en la Enseñanza de la Educación Física. Formación Inicial y Actualización. Un Estudio Exploratorio. Motricidad Humana., 12(1), 8-15.

Vera, J. A. (2010). Autobiografía, identidad docente y conocimiento didáctico en la enseñanza de la Educación Física. Revista Complutense de Educación, 21(2), 423-441.

Zabala, M. A. (2003). El aprendizaje experiencial como marco teórico para el prácticum. In Actas del VII Symposium Internacional sobre el Prácticum. (pp. 19-34). Pontevedra: Servicio de edición de la Universidad.

Zeng, H. Z., Leung, R. W., Liu, W., \& Bian, W. (2009). Learning outcomes tought by three teaching styles in college fundamental volleyball classes. Clinical Kinesiology, 63(1), 1-6.

Zhang, L. F. (2007). From career personality types to preferences for teachers' teaching styles: A new perspective on style mach. Personality and Individual Differences, (43), 18631874.

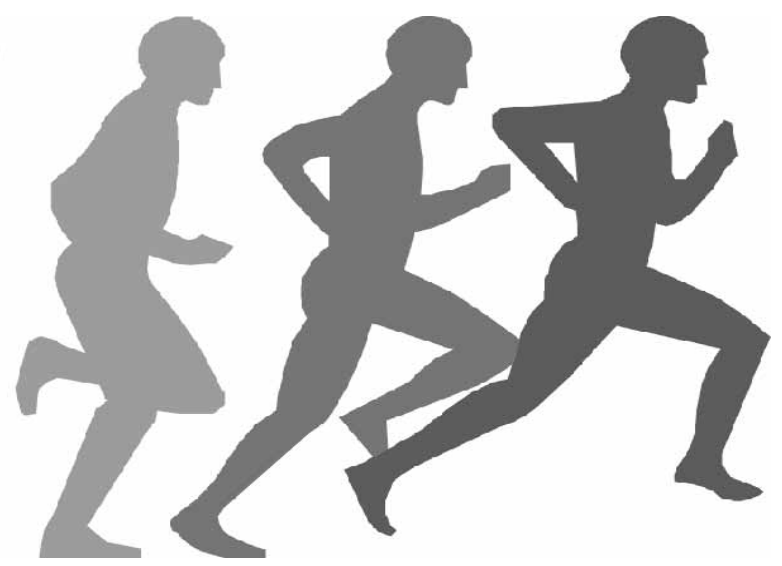

